\title{
Upregulation of Antioxidant Enzymes in Pancreatic Cancer Cells and Tissues
}

\author{
Simon G. Nyaga ${ }^{1}$, Frank Denaro ${ }^{1}$, Kaisha Hazel $^{2}$ and Blessing Akobundu ${ }^{3}$ \\ 1. Department of Biology, Morgan State University, Baltimore MD. \\ 2. Department of Radiology, Johns Hopkins University, Baltimore, MD. \\ ${ }^{3 .}$ Department of Molecular Pharmacology, Brown University, Providence, RI.
}

Pancreatic cancer (PC) is one of the deadliest forms of cancer. The incidence rate of PC is essentially equal to mortality rate primarily due to late diagnosis. The causes of PC are poorly understood, however, endogenous and exogenous Reactive Oxygen Species (ROS) such as hydroxyl radicals and hydrogen peroxide have been implicated as etiologic factors of the disease. Cancer cells have been shown to produce large amounts of hydrogen peroxide [1]. The interaction of ROS with cells causes oxidative damage to DNA and other macromolecules [2]. Unrepaired oxidative damage could cause mutations and cancer [3]. Cells harbor antioxidant enzymes that neutralize oxidants to minimize mutagenesis. We hypothesized that antioxidant enzymes may be upregulated in PC cells to counteract the deleterious effects of ROS. We therefore assessed the expression of the antioxidants: catalase (CAT), superoxide dismutase (SOD), and glutathione peroxidase1 (GPx1) in BxPC-3 PC cell line and PC tissues compared to negative and positive controls, immunohistochemically and by Western blot analyses.

The cell lines were cultured at $37^{\circ} \mathrm{C}$ and cell images captured using a 5 megapixel Android Camera on an inverted microscope (Zeiss) (Fig. 1, Panels: A and B). To assess GPx-1 expression in PC cells, cell-free extracts were prepared from the cancer and control cell lines. The total proteins were separated by SDSpolyacrylamide gel electrophoresis (SDS-PAGE) through 4-12\% step gradient gels. The protein bands were transferred electrophoretically onto a nitrocellulose membrane and the protein bands probed with primary and secondary antibodies and the protein bands developed using chemiluminiscence detection kit (Fisher Scientific). The protein bands were then detected autoradiographically. Immunohistochemical determination of CAT and SOD expression in PC tissues was screened on microarray tissue slides (Biomax Inc.) using CAT and SOD antibodies. A total of 24 PC samples including: ductal adenocarcinoma, adenosquamous carcinoma, neuroendocrine carcinoma, and controls (Fig. 3). The ABC technique was used immunocytochemically to label the samples and the images captured on a Nikon Microscope.

The cultured BxPC-3 cells formed distinct tumors (red arrows), whereas, the normal pancreatic cells formed uniform lawn of cells (blue arrow) (Fig 1A and Fig 1B, respectively). Western blot analysis showed that GPx1 was highly overexpressed in the PC cells relative to the control cells (Fig. 2). Immunohistochemical analysis of CAT showed that it was overexpressed in neuroendocrine tissues compared to the normal pancreatic tissues (Fig. 3, arrow 1, and Fig. 3 arrow 2, respectively). CAT was highly overexpressed in the positive control tissue (liver) (Fig. 3, arrow 3) as was expected due to high metabolic activity in that tissue. Histologic analysis showed that different cell types displayed high stain intensities for CAT and SOD within adenocarcinomas, neuroendocrine tumors, and in the liver cells (Fig. 4, Panels A, B and C, respectively). Altogether, these data are consistent with our hypothesis and suggest that pancreatic cancer cells have upregulated their antioxidant arsenals: CAT, SOD, and GPx1 possibly in response to increased ROS in PC cells. This response is essential in enhancing cellular survival due to the heavy insult of oxidatively-induced damage in cancer cells caused by ROS produced by increased 
metabolic reactions and as byproducts of cellular respiration. This research could lead to discovery of protein biomarkers useful in the control and prevention of PC as well as other forms of cancer [4].

\section{References:}

[1] Szatrowski, T.P. and Carl, F. Nathan., Cancer Research 51 (3) (1991), p. 794.

[2] Dizdaroglu, M., Free Radical Biology \& Medicine 10 (1991), p. 225.

[3] Ames, B. N., Shigenaga, M.K., and Hagen, T.M., PNAS 90 (17) (1993), p. 7915.

[4] The authors acknowledge financial support from ASCEND Grant (5UL1GM118973) and the Biology Department, Morgan State University.
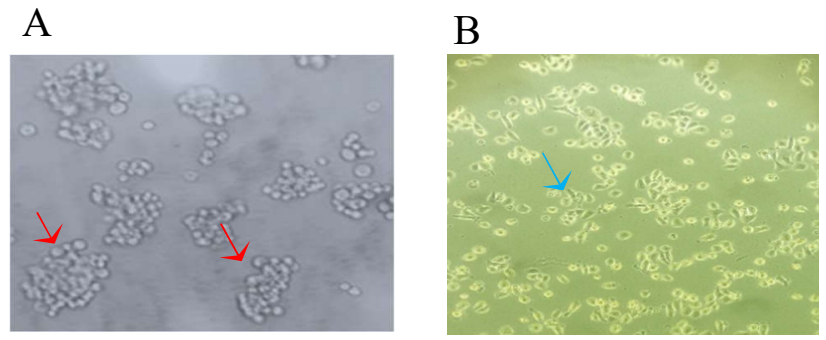

Figure 1. Microscopic images showing morphologies of cultured cells, Panel A, PC cells forming tumors (red arrows), and Panel B. normal PC cells forming uniform lawn of cells (blue arrow).

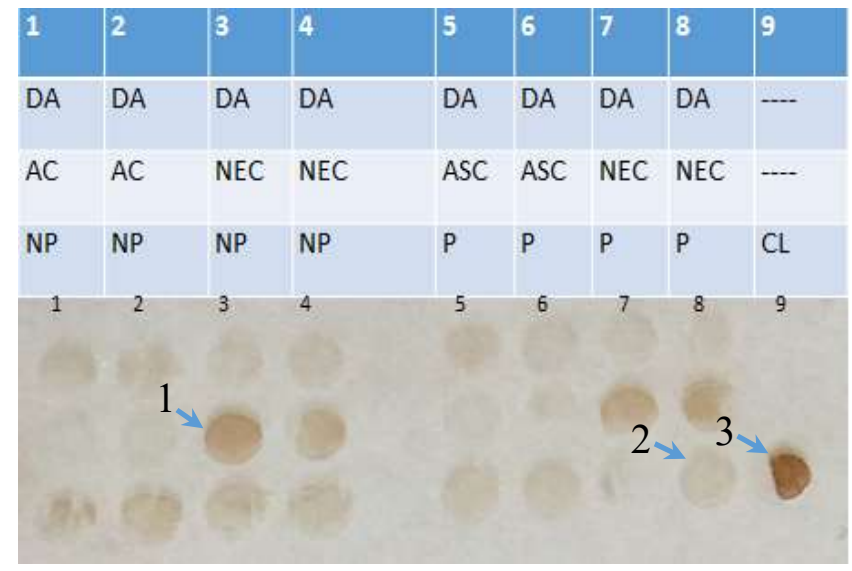

A

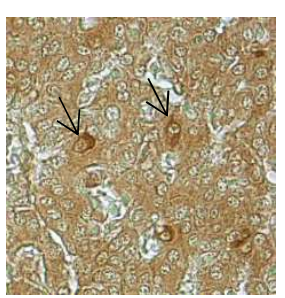

B

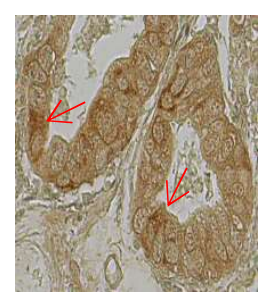

C

Figure 4. Immunohistochemistry of: A:

Adenocarcinoma tissue using anti-catalase antibodies, B: Neuroendocrine tissue using antisuperoxide dismutase antibodies, C: liver tissue (positive control) using anti-catalase antibodies. Arrows indicate distinctly staining cell types.

Figure 3. Immunohistochemical detection of catalase in pancreatic cancer tissue microarrays. DA, Ductal Adenocarcinoma; AC, Adenocarcinoma; NECNeuroendocrine Carcinoma; ASC, Adenosquamous Carcinoma; NP, Normal Pancreatic tissue; P, Pancreatic tissue; CL-Control Liver tissue (positive). The arrow labeled 1 shows overexpression of catalase in Neuroendocrine Carcinoma relative to Normal Pancreatic tissue (arrow 2), and arrow 3 shows expression of catalase in liver tissue (positive control). 